\title{
ANDES

www.scielo.cl

\section{Depleción electrolítica extrema asociada con shock séptico como forma de presentación de fibrosis quística}

\author{
Extreme electrolyte depletion associated with septic shock as a form of presentation \\ of cystic fibrosis
}

\author{
Camila Ampuero Acuña ${ }^{a}$, Gianfranco Tomarelli Rubio ${ }^{\text {, }}$, Soledad Montes Franceschini ${ }^{a}$, \\ Alejandro Donoso Fuentes ${ }^{a}$
}

aUnidad de Paciente Crítico Pediátrico, Hospital Clínico Dra. Eloísa Díaz I. La Florida. Santiago, Chile

Recibido: 27 de abril de 2021; Aceptado: 4 de agosto de 2021

¿Qué se sabe del tema que trata este estudio?

La deshidratación con alcalosis metabólica hipoclorémica es una manifestación conocida de la fibrosis quística, pero infrecuente como forma de presentación.
¿Qué aporta este estudio a lo ya conocido?

Se presenta un caso clínico que muestra una forma inusual y poco difundida de presentación de fibrosis quística. Se reporta como hallazgo de interés la combinación de dos variantes patogénicas en el gen CFTR, S945L y R1162X, que no habían sido relacionadas con la forma de presentación aquí comunicada.

\section{Resumen}

La deshidratación es una causa frecuente de consulta en emergencia pediátrica, siendo inhabitual la coexistencia de alcalosis metabólica hipoclorémica y deshidratación hiponatrémica. La presencia de alcalosis metabólica por depleción de cloro se ha reportado como una forma de presentación de Fibrosis Quística (FQ). Objetivo: Describir una forma de presentación inhabitual de fibrosis quística. Caso Clínico: Lactante masculino de 3 meses de edad quien presentó anormalidades del medio interno consistentes en alcalosis metabólica, hiponatremia, hipokalemia e hipocloremia extrema asociadas a shock séptico secundario a etiología mixta (Rhino/enterovirus, Streptoccus pneumoniae y Staphyloccus aureus). Se sospechó FQ, corroborándose el diagnóstico con medición de electrolitos en sudor y estudio genético, donde se evidenciaron dos variantes patogénicas; S945L (c.2834C>T o p.Ser945Leu) y R1162X (c.3484C >T o p.Arg1162X), ambas heterocigotas. Conclusión: Se debe prestar especial atención ante la existencia de alcalosis metabólica hipoclorémica y deshidratación hiponatrémica asociado o no a compromiso pulmonar, sospechando FQ como primera opción. Esta consideración toma mayor relevancia en aquellos países donde el test de tamizaje neonatal no está ampliamente disponible.
Palabras clave: Fibrosis Quística; Deshidratación; Hiponatremia; Hipokalemia; Déficit de Cloro; Alcalosis Metabólica adonosofuentes@gmail.com
Editado por:

Pablo Cruces Romero 


\begin{abstract}
In the pediatric emergency department, dehydrated children are one of the most frequent causes for consultation, however, the coexistence of hyponatremia with hypochloremia and metabolic alkalosis is rare. The presence of metabolic alkalosis due to chloride depletion has been reported as a form of presentation of Cystic Fibrosis (CF). Objective: to describe a case of cystic fibrosis of unusual presentation in a pediatric patient. Clinical Case: we report a 3-month-old previously healthy male infant who presented with internal environment abnormalities consisting of metabolic alkalosis, hyponatremia, hypokalemia, and extreme hypochloremia associated with septic shock due to mixed viralbacterial pneumonia (Rhino/enterovirus, Streptococcus pneumoniae, and Staphylococcus aureus). Cystic fibrosis (CF) was suspected, thus the diagnosis was corroborated by sweat test and genetic study which showed the pathogenic variants c.2834C $>\mathrm{T}$ (p.Ser945Leu) and c.3484C $>\mathrm{T}$ (p.Arg1162X), both heterozygous. Conclusion: special attention should be paid to the existence of hypochloremia with metabolic alkalosis and hyponatremia associated or not with pulmonary disease, suspecting CF as the first option. This consideration becomes more relevant in those countries where the neonatal screening test is not widely available.
\end{abstract}

Keywords:

Cystic Fibrosis;

Dehydration;

Hyponatremia;

Hypokalemia;

Chloride Deficiency;

Metabolic Alkalosis

\section{Introducción}

La fibrosis quística (FQ) es la enfermedad hereditaria autosómica recesiva más frecuente en la raza caucásica, con una incidencia de 1: 4.000 recién nacidos en EE.UU., pudiendo variar según el país ${ }^{1}$. En Chile se estima una incidencia de 1:8.000 a 10.000 recién nacidos vivos $^{2}$. La FQ se puede presentar con una amplia variedad de síntomas y signos, los cuales varían desde el período de recién nacido a la adultez. Su presentación clínica característica, más allá de la etapa neonatal, puede ser por manifestaciones respiratorias, digestivas, afectación hepática y pancreatobiliar, nasosinusal y anomalías urogenitales en pacientes de sexo masculino $^{3}$. En la actualidad, debido a la disponibilidad de tamizaje neonatal, se diagnostica con menor retraso ${ }^{1}$. En Chile este tamizaje está en etapa piloto realizándose en hospitales públicos de la Región Metropolitana y de la Región de Valparaíso.

Referente a los trastornos hidrolectrolíticos en el paciente con FQ estos pueden deberse a el tratamiento farmacológico empleado ${ }^{4} \mathrm{y}$ en ocasiones ser una manifestación de la misma enfermedad ${ }^{5}$. No obstante, la presencia de alcalosis metabólica por depleción de cloro puede ser su forma de presentación.

El objetivo de este reporte es presentar el caso de un paciente de tres meses de edad quién debutó con FQ al presentarse con alcalosis metabólica aguda, hiponatremia, hipokalemia e hipocloremia extrema, asociado con la presencia de shock séptico de foco pulmonar.

\section{Caso Clínico}

Lactante de 3 meses de edad, sexo masculino, sin antecedentes perinatales ni familiares de importancia.
Alimentado con leche materna exclusiva con buen incremento ponderal (aumento de 2,2 kg desde su nacimiento). Cursó la última semana de febrero (mes estival) con tos y rinorrea, afebril. Hubo exacerbación de sintomatología en últimos 3 días, agregándose decaimiento y rechazo alimentario. No presentó vómitos ni diarrea. Ingresó con temperatura de $38^{\circ} \mathrm{C}$, frecuencia cardíaca 124 lat/min, frecuencia respiratoria $40 \mathrm{resp} / \mathrm{min}$, presión arterial 100/68 $\mathrm{mmHg}$ (percentil 75), tiempo de llenado capilar en 4 segundos, saturación de oxígeno $88 \%$ y compromiso de conciencia. Al examen físico se evidenciaron signos de deshidratación y al examen pulmonar destacaron crépitos bilaterales. Se tomaron exámenes de laboratorio donde se observó alcalosis metabólica aguda con depleción electrolítica grave. Se complementó estudio con función renal, orina completa y electrolitos urinarios (tabla 1). El panel molecular respiratorio FilmArray resultó positivo para Rinovirus/Enterovirus. La radiografía de tórax reveló aumento del infiltrado intersticial bilateral con mayor confluencia hacia las bases, asociado a condensación en lóbulo inferior izquierdo en región retrocardiaca (figura 1).

Se ingresó a unidad de cuidados intensivos donde se efectuó intubación endotraqueal e inicio de ventilación mecánica. Evolucionó con shock séptico de foco pulmonar, recibiendo fluidos de reanimación (suero fisiológico al 0,9\%, $30 \mathrm{ml} / \mathrm{kg}$ en total), soporte vasoactivo con norepinefrina $(0,1 \mathrm{mcg} / \mathrm{kg} / \mathrm{min})$ y antibioticoterapia con cefotaxima, amikacina y cloxacilina dado sospecha de FQ en base al desbalance hidroelectrolítico y la sintomatología respiratoria presentada. Se indicó corrección electrolítica con cloruro de sodio $(\mathrm{NaCl})$ al $3 \%$ y cloruro de potasio $(\mathrm{KCl})$ al $10 \%$. Luego de 48 $72 \mathrm{~h}$ de iniciada la corrección se lograron valores normales de sodio y potasio plasmáticos, pudiendo ade- 
Tabla 1. Evolución temporal de los principales exámenes de laboratorio y cálculos derivados de paciente con debut de fibrosis quística

\begin{tabular}{|c|c|c|c|c|}
\hline Parámetro & Día 1 & Día 3 & Día 21 & 9 meses de evolución \\
\hline \multicolumn{5}{|l|}{ Electrolitos plasmáticos } \\
\hline $\mathrm{Na}(\mathrm{mEq} / \mathrm{L})$ & 117 & 137 & 137 & 138 \\
\hline $\mathrm{K}(\mathrm{mEq} / \mathrm{L})$ & 2,1 & 3,2 & 4,6 & 4,1 \\
\hline $\mathrm{Cl}(\mathrm{mEq} / \mathrm{L})$ & $<50$ & 99 & 105 & 108 \\
\hline $\mathrm{Ca}(\mathrm{mg} / \mathrm{dL})$ & 11,7 & - & 10,4 & - \\
\hline $\mathrm{Mg}(\mathrm{mg} / \mathrm{dL})$ & 1,9 & - & 2,0 & - \\
\hline$P(m g / d L)$ & 5,2 & - & 7,4 & - \\
\hline \multicolumn{5}{|l|}{ Gasometría arterial } \\
\hline $\mathrm{pH}$ & 7,61 & 7,37 & 7,35 & 7,38 \\
\hline $\mathrm{HCO}_{3}$ & 62,2 & 30,7 & 23,4 & 23,4 \\
\hline $\mathrm{PaCO}_{2}(\mathrm{mmHg})$ & 62,9 & 54 & 42,7 & 40,4 \\
\hline $\mathrm{BE}$ & 35,4 & 4,8 & $-2,0$ & $-1,5$ \\
\hline \multicolumn{5}{|l|}{ Función renal } \\
\hline $\mathrm{BUN}(\mathrm{mg} / \mathrm{dL})$ & 20 & 5 & 2 & 11 \\
\hline Creatinina (mg/dL) & 0,36 & 0,13 & 0,13 & 0,21 \\
\hline \multicolumn{5}{|l|}{ Análisis de orina } \\
\hline FENa (\%) & 8,9 & - & 0,03 & - \\
\hline $\mathrm{Cl}$ urinario $(\mathrm{mEq} / \mathrm{L})$ & 101 & 32 & 5,5 & $<20$ \\
\hline Proteinuria & +++ & ++ & negativo & negativo \\
\hline Hematuria (cel/uL) & 133 & 203 & 25 & $0-2$ \\
\hline Densidad urinaria & 1.025 & 1.012 & 1.015 & 1.009 \\
\hline \multicolumn{5}{|l|}{ Otros } \\
\hline $\mathrm{SvcO}_{2}(\%)$ & 49 & 73 & - & - \\
\hline Anion GAP & 5 & 7 & 9 & 7 \\
\hline DIFa (mEq/L) & 72 & - & 42 & - \\
\hline
\end{tabular}

Na: sodio; K: potasio; Cl: cloro; Ca: calcio; Mg: magnesio; P: fósforo; $\mathrm{HCO}_{3}$ : bicarbonato; $\mathrm{PaCO}_{2}$ : presión parcial de dióxido de carbono; BE: exceso de base; BUN: nitrógeno ureico sanguíneo; FENa: fracción excretada de sodio; $\mathrm{Cl}$ urinario: cloro urinario; SvcO ${ }_{2}$ : saturación venosa central de oxígeno; DIFa: diferencia de iones fuertes aparente; $\mathrm{mEq} / \mathrm{L}$ : miliequivalentes por litro; mg/dL: miligramos por decilitro; $\mathrm{mmHg}$ : $\mathrm{mi}-$ límetros de mercurio; cel/uL: células por microlitro.

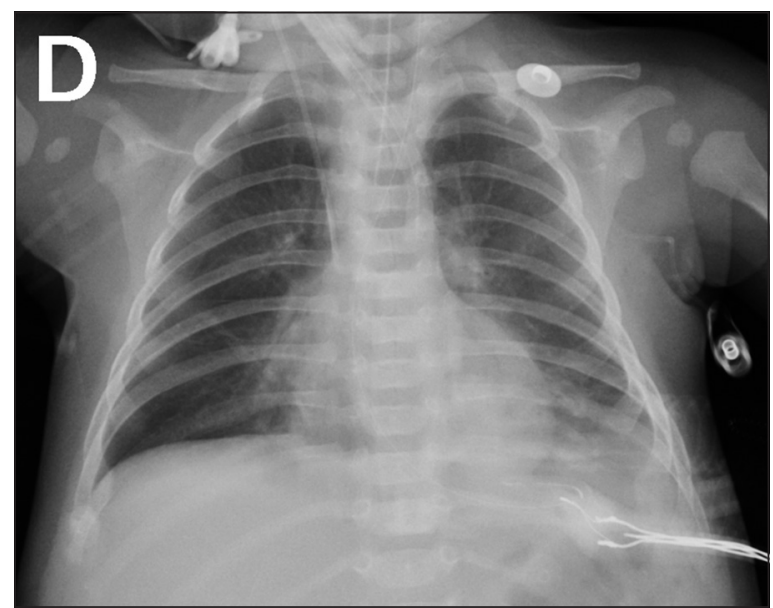

Figura 1. Radiografía de tórax anteroposterior que revela aumento del infiltrado intersticial bilateral con mayor confluencia hacia las bases, asociado a condensación en lóbulo inferior izquierdo en región retrocardiaca. más extubarse satisfactoriamente. Se realizó ecografía abdominal que no evidenció estenosis hipertrófica del píloro ni signos de nefrocalcinosis. Cursó con atelectasia en lóbulo superior derecho de difícil manejo, requiriendo nebulizaciones con solución hipertónica al $7 \%$ y kinesioterapia respiratoria intensiva. Se rescataron los cultivos tomados a su ingreso, donde destacó cultivo de aspirado traqueal positivo para Streptococcus pneumoniae sensible a cefalosporinas y Staphylococcus aureus multisensible, por lo que se trató con cefotaxima y cloxacilina por 7 días y amikacina por 5 días, completando posteriormente 10 días de antibiótico con cotrimoxazol vía oral.

Se realizó prueba del sudor en dos oportunidades. Inicialmente, el cloro en sudor fue de 56 $\mathrm{mmol} / \mathrm{L}$ (sospecha), mientras que la segunda prueba resultó con un valor de $65 \mathrm{mmol} / \mathrm{L}$ lo cual permitió confirmar el diagnóstico. Se realizó medición 
Fibrosis Quística - C. Ampuero Acuña et al

de los niveles de elastasa pancreática en materia fecal que resultó > $500 \mathrm{ug} / \mathrm{g}$ heces $(\mathrm{VN}>200 \mathrm{ug} / \mathrm{g}$ ). El estudio del gen de la proteína reguladora de la conducción transmembrana (CFTR, Cystic fibrosis transmembrane conductance regulator) (análisis de la secuencia y prueba de deleción/duplicación del gen CFTR, Laboratorio Invitae) evidenció dos variantes patogénicas; c.2834C > T (p.Ser945Leu) y c.3484C > T (p.Arg1162X), ambas heterocigotas. El paciente fue dado de alta en buenas condiciones con sodio suplementario, vitaminas liposolubles, nebulizaciones con suero hipertónico y kinesioterapia respiratoria. Ingresó al programa nacional de FQ. En el seguimiento al año de evolución el paciente se encuentra colonizado en forma crónica por Staphylococcus aureus, con exacerbaciones respiratorias que se han logrado manejar en forma ambulatoria, presentando además un adecuado incremento pondoestatural.

\section{Discusión}

Este caso muestra una forma poco frecuente de presentación de FQ caracterizada por alcalosis metabólica, hiponatremia, hipokalemia e hipocloremia. Los factores habitualmente asociados a su desarrollo en el paciente con FQ son: presentar una edad menor de un año, existencia de deshidratación, exposición reciente a una temperatura ambiental elevada, incremento de la temperatura corporal e infección respiratoria intercu- rrente $^{6}$. Además, en lactantes, la alimentación materna exclusiva o con fórmulas con bajo contenido de sodio puede ser insuficiente para cubrir sus requerimientos ${ }^{7}$. Todos estos factores clínicos y ambientales previamente mencionados se pueden identificar en el caso aquí reportado.

En condiciones normales el cloro se reabsorbe del sudor por medio de la proteína CFTR, ocasionando también la reabsorción del sodio, dado la activación de los canales ubicados en las células ductales. En los pacientes con FQ la producción primaria de sudor es relativamente normal, sin embargo, la existencia de una deficiente absorción de cloro origina una falla en la activación de los canales de sodio y ante escenarios que originen una sudoración excesiva se excreta una gran cantidad de $\mathrm{NaCl}$ y por consiguiente pérdida de estos electrolitos como también de fluidos por esta vía. Esto causa activación del sistema simpático, incremento en la liberación de la hormona antidiurética y activación del eje renina- angiotensina-aldosterona con desarrollo de hiperaldosteronismo secundario, ocasionando reabsorción de sodio y agua y excreción de potasio e hidrogeniones en el túbulo colector cortical renal; además la depleción de cloro origina alcalosis metabólica por reabsorción renal de bicarbonato ${ }^{8}$ (figura 2). Estos fenómenos reciben en la actualidad el nombre de alcalosis metabólica por depleción de cloro, permitiendo destacar así el rol trascendental de la falta del cloro en su origen ${ }^{9}$.

En el paciente deshidratado con alcalosis meta-

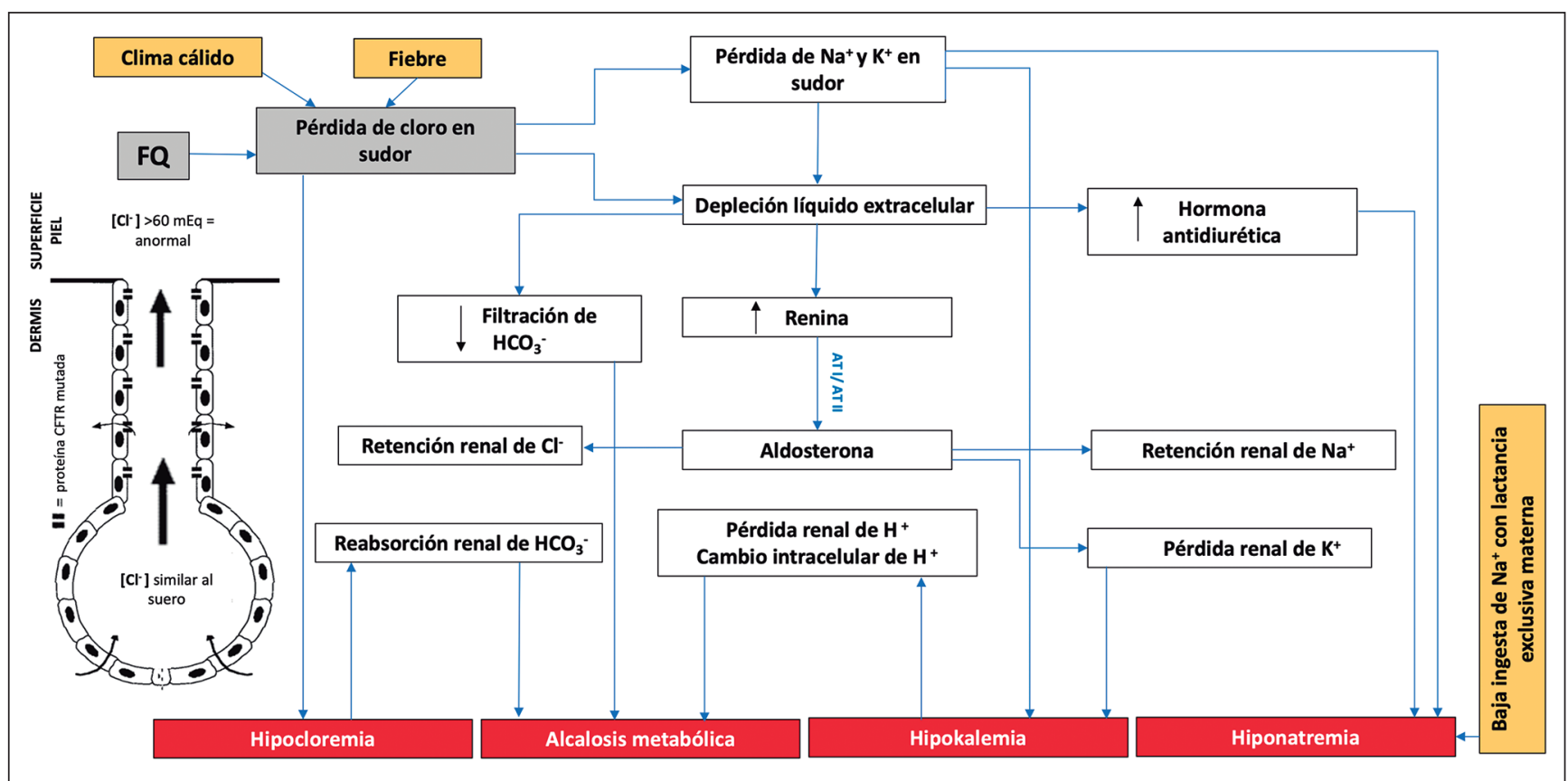

Figura 2. Mecanismos fisiopatológicos involucrados en el desarrollo de la depleción electrolítica en el paciente con fibrosis quística. No se muestra en la figura el efecto de los vómitos y diarrea, como tampoco de una eventual reducción de la función renal. FQ: fibrosis quística; CFTR: proteína reguladora de la conducción transmembrana; Cl: cloro; Na: sodio; K: potasio; HCO3: bicarbonato; AT I/AT II: angiotensina I y II; H:hidrogeniones. 
bólica hipoclorémica, es importante mencionar entre los principales diagnósticos a evaluar a la estenosis hipertrófica del píloro donde se produce alcalosis por la pérdida de hidrogeniones por vómitos, sin embargo en esta patología no se presenta hipokalemia ni hiponatremia. El síndrome de Bartter obedece a una alteración congénita en la reabsorción tubular de cloro (mutación de la proteína del canal iónico de cloro- $\mathrm{Kb}$ ), por lo que a diferencia del paciente con $\mathrm{FQ}$ aquí las pérdidas de cloro son de origen renal. Este se caracteriza por presentar además de las alteraciones de laboratorio previamente mencionadas, hipokalemia grave e hiperreninemia. Otras condiciones más infrecuentes de observar y las cuales son catalogadas como síndrome de pseudo-Bartter dada la similitud bioquímica, son la diarrea perdedora de cloruros, las pérdidas gástricas continuas sin una adecuada reposición y el uso excesivo o mal uso de diuréticos de asa ${ }^{10}$. En nuestro paciente no existieron pérdidas por diarrea o vómitos intercurrentes, lo que nos permite destacar el rol de la excesiva pérdida electrolitos por el sudor para causar tan extrema pérdida de sal.

En el caso aquí descrito, en un inicio y en forma transitoria la medición de cloro en orina fue elevada, lo que podría ser explicado por la presencia de disfunción tubular renal transitoria ocasionada por el episodio séptico en curso $^{11}$, observándose posteriormente una disminución del cloro urinario a valores menores 20 $\mathrm{mEq} / \mathrm{L}$ en concomitancia con la normalización de la función renal. Además, existió una respuesta favorable a la administración de cloro mediante solución salina, reflejando la existencia de alcalosis metabólica de carácter cloro-sensible, lo que orienta al origen extrarrenal de estas pérdidas ${ }^{12}$.

Referente a las causas y signos clínicos de depleción de líquidos en los niños con FQ es posible que en los lactantes pueda existir una subestimación por parte de los padres en lo referente a las reales necesidades de líquidos a aportar, experimentando deshidratación ante una exposición prolongada a un clima cálido, lo que a su vez origina una mayor pérdida de sodio y cloro por el sudor. La hipocloremia prácticamente no presenta manifestaciones clínicas específicas, aunque con niveles de cloro bajo $85 \mathrm{mmol} / \mathrm{L}$ se ha descrito el desarrollo de hipotensión arterial. Por otra parte, en los pacientes depletados de sal, los signos físicos tradicionales de deshidratación son menos evidentes. Finalmente, en ocasiones en las cuales se ha medido sodio urinario en pacientes clínicamente estables, este se encuentra disminuido, estando asociado a niveles elevados de renina y aldosterona ${ }^{13}$, todo lo cual nos sugiere la presencia de una condición crónica de pérdida de fluidos corporales en estos pacientes.

Las descripciones que alertaron sobre esta forma de presentación para la FQ fueron las de Rendle-Short ${ }^{14}$,
Di Sant 'Agnese ${ }^{15}$ y Nussbaum ${ }^{16}$ entre otros, siendo este último quién reportó a dos lactantes, quienes representaron menos del $1 \%$ de los pacientes de este rango etáreo durante un período de dos décadas. En la casuística de Sojo $^{17}$ donde se evaluó la existencia de déficit de cloro y alcalosis metabólica en pacientes con $\mathrm{FQ}$, en un $38 \%$ esta fue su forma de presentación. Por otra parte, en una comunicación más reciente de Ballestero ${ }^{10}$, se describió una prevalencia de alcalosis metabólica por depleción de sodio y cloro como presentación de FQ de $16,8 \%$.

En comparación con un caso nacional publicado hace tres décadas ${ }^{18}$ donde se describe un lactante de tres meses de edad, este presentaba una historia previa de episodios de neumonia y atelectasia antes de establecerse el diagnóstico definitivo. No obstante, aunque la sospecha y el diagnóstico es cada vez más temprano para los pacientes con FQ dado la estrategia de pesquiza neonatal, aún en ocasiones este se establece luego de varios episodios de deshidratación ${ }^{10} \mathrm{o}$ infección respiratoria ${ }^{8} \mathrm{y}$ a pesar de haber tenido contacto previo con el equipo de salud. El retraso en reconocer esta condición clínica referente a su forma de presentación resultará en un retardo en su diagnóstico y por consiguiente repercusión en el pronóstico nutricional y respiratorio.

Una contundente pista diagnóstica fue el hallazgo de Staphylococcus aureus en el cultivo de secreción traqueal. Las secreciones bronquiales más adherentes de los pacientes con FQ, facilita la colonización y eventual infección por gérmenes menos habituales. Las infecciones pulmonares por Staphylococcus aureus, Pseudomona aeruginosa, Stenotrophomona maltophilia y Burkhorderia cepacia ${ }^{2}$, entre otros, son características en este grupo de pacientes y su hallazgo en un paciente sin diagnóstico de FQ deben ser un signo de alarma de la enfermedad de base, recomendándose su tratamiento precoz y agrevivo para disminuir el daño pulmonar crónico y mejorar la sobrevida.

Se han descubierto más de 2000 mutaciones para el gen CFTR, las cuales se dividen en cinco tipos distintos, en función de los efectos fisiopatológicos producidos en la proteína para el desarrollo de la enfermedad. La forma de presentación aquí señalada se ha asociado a mutaciones del gen CFTR que preservan parcialmente su funcionalidad, siendo esta superior al $10 \%$ de la actividad de la proteína CFTR, asociándose con manifestaciones fenotípicas más leves ${ }^{8}$. La mutación con mayor frecuencia involucrada en esta forma de presentación es la F508del ${ }^{19}$. Otras mutaciones descritas son T338I, D110E y D110H ${ }^{20-22}$. En nuestro paciente fueron identificados dos variantes patogénicas en el gen CFTR, S945L (p.Ser945Leu o c.2834C >T) y R1162X (p.Arg1162X o c.3484C > T), combinación que causa la enfermedad. Esta asociación de variantes es infrecuente, existiendo solo un paciente registrado en la 
base de datos CFTR2 ${ }^{23}$ no pudiéndose así establecer la relación de esta combinación con la forma de presentación aquí comunicada. La mutación R1162X es una mutación de clase $\mathrm{I}^{24}$ resultando en una expresión de la proteína CFTR gravemente reducida o ausente. La mutación S945L es considerada una combinación de mutación clase II-III ${ }^{25}$ resultando en una disminución en la cantidad y/o función de la proteína CFTR, pero con una función residual que parece ser suficiente para evitar las manifestaciones clínicas típicas de la FQ. Así es como, ante la sospecha de FQ, es fundamental establecer la suficiencia pancreática, mediante la determinación de elastasa pancreática en materia fecal, siendo fundamental la suplementación enzimática en caso de insuficiencia para lograr un adecuado desarrollo pondoestutural lo que finalmente incide en el pronóstico pulmonar a mediano y largo plazo $^{2}$. En este caso, el paciente presentaba suficiencia pancreática no siendo necesaria la suplementación enzimática, sin embargo, se debe realizar seguimiento nutricional y medición de elastasa seriada ya que a mediano o largo plazo se puede agregar una insuficiencia pancreática ${ }^{2}$.

Referente al tratamiento, ante la existencia de deshidratación y los trastornos electrolíticos previamente mencionados la reposición debe ser por vía parenteral, salvo que se trate de casos leves donde se puede utilizar la rehidratación oral. Como lo aquí comunicado, habitualmente las anormalidades del sodio, cloro y potasio y la alcalosis metabólica mejoran significativamente con la terapia empleada.

Finalmente, es importante destacar que los lactantes con FQ no diagnosticados en forma oportuna corren el riesgo de desarrollar esta grave deficiencia electrolítica con alcalosis metabólica y eventuales complicaciones neurológicas y cardíacas ${ }^{3}$. Esto toma especial relevancia en los países que carecen de un programa de tamizaje neonatal ${ }^{1}$.

\section{Conclusión}

El médico debe de estar alerta que las anormalidades electrolíticas aquí descritas pueden ocurrir como forma de presentación de fibrosis quística, aún en ausencia de los signos clásicos de la enfermedad. Esta consideración toma mayor relevancia en aquellos países donde el test de tamizaje neonatal no está ampliamente disponible.

\section{Responsabilidades Éticas}

Protección de personas y animales: Los autores declaran que los procedimientos seguidos se conformaron a las normas éticas del comité de experimentación humana responsable y de acuerdo con la Asociación Médica Mundial y la Declaración de Helsinki.

Confidencialidad de los datos: Los autores declaran que han seguido los protocolos de su centro de trabajo sobre la publicación de datos de pacientes.

\section{Derecho a la privacidad y consentimiento informado: Los autores han obtenido el consentimiento informa- do de los pacientes y/o sujetos referidos en el artículo. Este documento obra en poder del autor de correspon- dencia referidos en el artículo. Este documento obra en poder del autor de correspondencia}

\section{Conflicto de intereses}

Los autores declaran no tener conflicto de intereses.

\section{Referencias}

1. Farrell P, White T, Ren C, et al. Diagnosis of Cystic Fibrosis: Consensus Guidelines from the Cystic Fibrosis Foundation. J Pediatr. 2017;181S:S4-S15.e1.

2. Grupo Técnico, Programa de Fibrosis Quística, Departamento Procesos Clínicos Integrados, Subsecretaría de Redes asistenciales. ORIENTACIONES TÉCNICAS PARA LA ATENCIÓN INTEGRAL DE FIBROSIS QUÍSTICA. Ministerio de Salud, Gobierno de Chile. 2da edición 2019. https://www. minsal.cl/wp-content/uploads/2019/08/ ORIENTACIONES-T\%C3\%89CNICASFIBROSIS-QUISTICA-2019.pdf , última visita en marzo 2021.

3. Yalçin E, Kiper N, Doğru D, Özçelik Aslan A. Clinical features and treatment approaches in cystic fibrosis with pseudo-
Bartter syndrome. Ann Trop Paediatr. 2005;25(2):119-24.

4. Liamis G., Milionis H., Elisaf M. A review of drug-induced hyponatremia. Am J Kidney Dis. 2008;52(1):144-53.

5. Kerem E. Atypical CF and CF related diseases. Paediatr Respir Rev. 2006;7 Suppl 1:S144-6.

6. Scurati-Manzoni E., Fossali E., Agostoni C. et al. Electrolyte abnormalities in cystic fibrosis: systematic review of the literature. Pediatr Nephrol. 2014;29(6):1015-23.

7. Coates AJ., Crofton PM., Marshall T. Evaluation of salt supplementation in CF infants. J Cyst Fibros. 2009;8(6):382-5.

8. Aranzamendi R, Breitman F, Asciutto C, Delgado N, Castaños C. Deshidratación con alcalosis hipoclorémica: presentación inusual de fibrosis quística en un lactante. Arch Argent Pediatr 2008;106(5):443-457.
9. Luke RG, Galla JH. It is chloride depletion alkalosis, not contraction alkalosis. J Am Soc Nephrol. 2012;23(2):204-7.

10. Ballestero Y, Hernandez M, Rojo P, et al. Hyponatremic Dehydration as a Presentation of Cystic Fibrosis. Pediatr Emerg Care. 2006;22(11):725-7.

11. Anderson RJ, Gabow PA, Gross PA. Urinary chloride concentration in acute renal failure. Miner Electrolyte Metab. 1984;10(2):92-7.

12. Mesa O, González JL, García V, Romero S, Marrero C. Alcalosis metabólica de origen dietético en un lactante. An Pediatr (Barc). 2009;70(4):370-3.

13. Stenvinkel P, Hjelte L, Alván G, Hedman A, Hultman E, Strandvik B. Decreased renal clearance of sodium in cystic fibrosis. Acta Paediatr Scand. 1991;80(2):194-8.

14. Rendle-Short J. Fibrocystic disease 
of the pancreas presenting with acute salt depletion. Arch Dis Child. 1956;31(155):28-30.

15. Di Sant 'Agnese P. Salt depletion in cold weather in infants with cystic fibrosis of the pancreas. J Am Med Assoc. 1960;172:2014-21.

16. Nussbaum E, Boat T, Wood R, Doershuk C. Cystic fibrosis with acute hypoelectrolytemia and metabolic alkalosis in infancy. Am J Dis Child. 1979;133(9):965-6.

17. Sojo A, Rodriguez-Soriano J, Vitoria JC, Vasquez C, Ariceta G, Villate A. Chloride deficiency as a presentation or complication of cystic fibrosis. Eur J Pediatr. 1994;153(11):825-8.

18. Param T, Aldunate D. Metabolic alkalosis in cystic fibrosis of the pancreas. Rev Chil Pediatr. 1988;59(5):322-5.

19. Fustik S, Pop-Jordanova N, Slaveska N, Koceva S, Efremov G. Metabolic alkalosis with hypoelectrolytemia in infants with cystic fibrosis. Pediatr Int. 2002;44(3):28992.

20. Battista Leoni G, Pitzalis S, Podda R, et al. A specific cystic fibrosis mutation (T338I) associated with the phenotype of isolated hypotonic dehydration. J Pediatr. 1995;127(2):281-3.

21. Padoan R, Bassotti A, Seia A, et al. A novel missense mutation (D110E) in exon 4 of CFTR (ABCC7) in a CF infant presenting with hypochloraemic metabolic alkalosis. Hum Mutat. 2000;15(5):485.

22. Weller F, Wiebicke W, Tümmler B.
Turkish infant with hypoelectrolytemia and metabolic alkalosis as the sole manifestations of a mild form of cystic fibrosis (mutation D110H). Klin Padiatr. 2000;212(1):41-3.

23. Clinical and Functional Translation of CFTR2. Portal [Internet], https://www. cftr2.org/, última visita en marzo 2021

24. Murieli K, da Silveirea E, Correia C, et al. Genetic and phenotypic traits of children and adolescents with cystic fibrosis in Southern Brazil. J Bras Pneumol. 2018;44(6):498-504.

25. Veit G, Avramescu R, Chiang A, et al. From CFTR biology toward combinatorial pharmacotherapy: expanded classification of cystic fibrosis mutations. Mol Biol Cell. 2016;27(3):424-33. 